\title{
Development and Characterisation of Disulfiram-loaded PLGA Nanoparticles for the Treatment of Non-Small Cell Lung Cancer
}

Mohammad Najlah ${ }^{1}$, Zahima Ahmed ${ }^{2}$, Mohammed lqbal ${ }^{2}$, Zhipeng Wang ${ }^{3}$, Patrica Tawari $^{3}$, Weiguang Wang ${ }^{3}$ and Christopher McConville ${ }^{4 *}$

${ }^{1}$ Department, Medicine and Healthcare Science, Faculty of Medical Science, Anglia Ruskin University, Chelmsford, Essex, CM1 1SQ.

${ }^{2}$ School of Pharmacy, Faculty of Science and Engineering, University of Wolverhampton, Wulfrana Street, Wolverhampton WV1 1LY, UK

${ }^{3}$ Research Institute in Healthcare Science, Faculty of Science and Engineering, University of Wolverhampton, Wulfrana Street, Wolverhampton WV1 1LY, UK ${ }^{4}$ School of Pharmacy, Institute of Clinical Sciences, College of Medical and Dental Sciences, University of Birmingham, Edgbaston, B15 2TT.

${ }^{*}$ Corresponding author. Tel.: 01214143209

E-mail address: C.Mcconville.2@bham.ac.uk

Running Title: Development and Characterisation of Disulfiram-loaded PLGA Nanoparticles for the Treatment of Non-Small Cell Lung Cancer

\section{Keywords}

Disulfiram, Lung Cancer, PLGA, Nanoparticles 


\section{Abstract}

Non-Small Cell Lung Cancer (NSCLC) is the most common type of lung cancer in both men and women. A recent phase llb study demonstrated that disulfiram (DSF) in combination with cisplatin and vinorelbine was well tolerated and prolonged the survival of patients with newly diagnosed NSCLC. However, DSF is rapidly (4 minutes) metabolised in the bloodstream and it is this issue which is limiting its anticancer application in the clinic. We have recently demonstrated that a low dose of DSF-loaded PLGA nanoparticles supplemented with oral $\mathrm{Cu}$ inhibited tumour growth and reduced metastasis in a xenograft mouse lung cancer model. Here we demonstrate the influence of PLGA polymer, stabilizer loading and molecular weight as well as sonication time on the characteristics, including DSF release and the cytotoxicity of $10 \% \mathrm{w} / \mathrm{w}$ DSF-loaded PLGA nanoparticles. The paper demonstrates that the choice of PLGA as no significant influence on the characteristics of the nanoparticles apart from their DSF release, which is due to the differing degradation rates of the polymers. However, increasing the loading and molecular weight of the stabilizer as well as the sonication time reduced the size of the nanoparticles, reduced their ability to protect the DSF from reacting with $\mathrm{Cu}$ and degrading in serum, while increasing their DSF release rate and cytotoxicity. Additionally, increasing the sonication time resulted in the premature degradation of the PLGA, which increased the permeability of the nanoparticles further decreasing their ability to protect DSF from reacting with $\mathrm{Cu}$ and degrading in serum, while increasing their DSF release rate and cytotoxicity. 


\section{Introduction}

Lung cancer is the second most common cancer in both men and women behind prostrate and breast cancer respectively [1]. There were more than 1.8 million new cases (13\% of total cancer incidence) and almost 1.6 million deaths ( $20 \%$ of total cancer mortality) globally [1]. Lung cancer is categorised into two types, non-small cell lung cancer (NSCLC) and small cell lung cancer (SCLC), with NSCLC accounting for $87 \%$ of lung cancer cases [2]. The prognosis for NSCLC patients is poor with only $15 \%$ of patients surviving longer than 5-years and a high rate of recurrence [3-5].

Current treatment options for NSCLC are radiotherapy and chemotherapy, both which have their limitations such as toxicity, serious systemic side-effects, low selectivity and resistance [6]. The systemic administration of chemotherapeutic drugs has its limitations due to the need for higher dose, which result in serious adverse side-effects as these drugs destroy both cancerous and healthy cells [7-8]. Encapsulating a chemotherapeutic drug in a nanoparticle offers a number of advantages, such as protection from degradation in the blood stream, improved drug solubility, targeted drug delivery, decreased side-effects, increased drug exposure time and reduced drug resistance. Nanoparticles tend to be manufactured using biodegradable polymers like poly(lactic-co-glycolic acid) (PLGA) or polycaprolactone (PCL) and have been shown to have increased efficacy and reduced toxicity compared to conventional delivery of chemotherapeutic drugs [9]. If designed correctly nanoparticle formulations can be made to accumulate in cancerous tissue by either passive or active targeting without being excreted from the body [10]. The small size of the nanoparticles allows them to accumulate in tumours by extravasation due to leaky vasculature and a poorly developed lymphatic drainage 
system [11-12]. Poorly designed nanoparticles will be taken up by the reticuloendothelial system (RES) of the body. Nanoparticles with a hydrophobic surface are rapidly taken up by the liver, spleen and lungs [13], while those with a hydrophilic surface showed an increased circulation time in the body [14]. Therefore, to achieve increased circulation time in the bloodstream and thus improved targeting, nanoparticles need to be $100 \mu \mathrm{M}$ or less is size with a hydrophilic surface in order to reduce clearance by macrophages [15]. Paclitaxel nanoparticles have been shown to have similar efficacy, with faster administration times, when compared to conventional formulations in preclinical studies [16-17]. Doxorubicin-loaded PLGA nanoparticles where targeted to A549 lung cancer cells by the attachment of a CXCR4 antagonist (LFC131 peptide) to their surface [18].

NSCLC and other cancers contain a small population of Cancer Stem Cells (CSCs) which are resistant to radio- and chemotherapy and are responsible for cancer recurrence [19-20]. Therefore the discovery of a CSC-targeting drug would significantly improve chemotherapy outcomes for NSCLC patients. The antialcoholism drug disulfiram (DSF) has been shown to have an anticancer effect against prostate cancer, breast cancer, brain tumours, leukaemia, cervical adenocarcinoma and NSCLC [21-34]. Furthermore, DSF has been shown to be an irreversible aldehyde dehydrogenase (ALDH) inhibitor targeting CSCs [22, 35-36]. This anticancer effect is copper (Cu) dependent [37-38] as $\mathrm{Cu}$ plays a crucial role in redox reactions which trigger the generation of reactive oxygen species (ROS) inducing apoptosis in human cells [39]. A recent phase Ilb study demonstrated that DSF in combination with cisplatin and vinorelbine was well tolerated and prolonged the survival of patients with newly diagnosed NSCLC [40]. DSF chelates Cu(II) forming a DSF/Cu complex increasing the transport of $\mathrm{Cu}$ into cancer cells and is a 
much stronger ROS inducer than $\mathrm{Cu}$ alone [41-42]. Drug induced ROS accumulation can be counterbalanced by the activation of NFKB, which inhibits ROS and ROS-induced cytotoxicity [43] but DSF inhibits the activity of NFKB [44]. For this anticancer effect to occur to DSF must accumulate in the cancer cell in its unaltered form and chelate with copper in the cancer cell. However, DSF is rapidly (4 minutes) metabolised in the bloodstream and it is this issue which is limiting its anticancer application in the clinic. Therefore, a drug delivery system which protects DSF from metabolism in the blood stream and targets it to NSCLC cells would expedite translation of DSF into the clinic as a treatment for NSCLC. To this end, we recently formulated DSF into liposomes, PLGA and gold nanoparticles, which at very low dose in combination with an oral $\mathrm{Cu}$ supplement demonstrated a significant tumour inhibiting effect in xenograft mouse models of breast and lung cancer [36, 45]. Furthermore, we also recently demonstrated that PLGA nanoparticles can extend the half-life of DSF in blood from under 2 minutes to 7 hours. The DSF-loaded PLGA nanoparticles in combination with copper significantly inhibited the liver CSC population and demonstrated very promising anticancer efficacy and anti-metastatic effect in a liver cancer mouse model [46].

In this study we will investigate the influence of the PLGA type, sonication time, the amount and molecular weight of stabiliser on the morphology, particle size, zeta potential, encapsulation efficiency, drug release, the ability to protect DSF and cytotoxicity against lung cancer of DSF-loaded PLGA nanoparticles. 


\section{Materials and Methods}

\subsection{Materials}

Poly (D,L-lactide-co-glycolide) (PLGA) was purchased from Evonik (Birmingham, AL, USA), while Polyvinyl acetate (PVA), Disulfiram (DSF), Dichloromethane (DCM), Tween 80, Sucrose, Phosphate-buffered saline (PBS) ( $\mathrm{pH} 7.4)$, Methanol and Copper (Cu), were all purchased from Sigma-Aldrich (Dorset, UK).

\subsection{Preparation of the $10 \%$ w/w DSF-loaded PLGA nanoparticles}

10\% w/w DSF loaded PLGA nanoparticles were prepared using the emulsion-solvent evaporation method. $50 \mathrm{mg}$ of DSF and $450 \mathrm{mg}$ of PLGA were dissolved in $10 \mathrm{ml}$ of DCM. $100 \mathrm{mg}$ of PVA was dissolved in $25 \mathrm{ml}$ of water at $40^{\circ} \mathrm{C}$ using a hotplate stirrer and the PLGA/DSF solution added drop wise using a burette with an 18G needle and left to stir for 30 minutes. The subsequent solution was probe-sonicated 9 times for 3 minutes at $60 \%$ power, and left to stir at $40^{\circ} \mathrm{C}$ overnight to remove the residual DCM. The nanoparticle solutions were centrifuged at $9500 \mathrm{rpm}$ for 20 minutes and the supernatant removed. The pellets were re-suspended in $25 \mathrm{ml}$ of distilled water and centrifuged and the supernatant removed. This wash-step was repeated one more time. Finally the washed nanoparticles were suspended in $10 \mathrm{ml}$ of $1 \%$ sucrose and placed in the freezer overnight to freeze. The frozen solution was freeze dried for 72 hours, with the primary drying stage lasting 48 hours at 0.5 millibar and the secondary drying stage 24 hours at 0.35 millibar. The dried nanoparticles were stored in the fridge until required. The above procedure was repeated with the type of PLGA, sonication time, amount and molecular with of PVA varied as per table 1. 


\subsection{Morphology and size of the $10 \%$ w/w DSF-loaded PLGA nanoparticles}

Scanning Electron Microscopy (SEM) images of the $10 \% \mathrm{w} / \mathrm{w}$ disulfiram loaded PLGA nanoparticles where taken using a Zeiss EV050-EP scanning electron microscopy. A small amount of each nanoparticle sample was placed onto an aluminium stub using an adhesive carbon tab then sputter-coated with gold.

\subsection{Particle size and zeta potential of the $10 \%$ W/W DSF-loaded PLGA Nanoparticles}

Particle size and zeta potential were measured by photon correlation spectroscopy using a Zetasizer HAS 3000 from Malvern (Worecestershire, UK). $100 \mu \mathrm{L}$ of the nanoparticle suspension was dispersed in $4 \mathrm{~mL}$ deionized water and sonicated for 1 min. Measurements were carried out at $25^{\circ} \mathrm{C}$.

\subsection{Encapsulation efficiency of the $10 \%$ w/w DSF-loaded PLGA nanoparticles}

Encapsulation efficiency was determined by dissolving $5 \mathrm{mg}$ of each nanoparticle formulation $(n=5)$ in DCM. The DCM was evaporated overnight and the residue dissolved in ethanol. The ethanol was filtered (pore size $0.22 \mu \mathrm{m}$, Millipore) and subsequently analysed using the stability indicating DSF HPLC method.

\subsection{In- vitro release of the $10 \%$ W/W DSF-loaded PLGA nanoparticles}

Each $10 \% \mathrm{w} / \mathrm{w}$ disulfiram loaded PLGA nanoparticle formulation $(\mathrm{n}=5)$ was placed into a dialysis bag containing $3 \mathrm{ml}$ of $1 \% \mathrm{w} / \mathrm{v}$ tween solution. The dialysis bag was subsequently placed into a glass beaker containing $22 \mathrm{ml}$ of $1 \% \mathrm{w} / \mathrm{v}$ tween solution. $300 \mu \mathrm{L}$ samples were taken once every day for 7 days and replaced with $300 \mu \mathrm{L}$ of fresh $1 \%$ tween. The samples were subsequently analysed using the stability indicating DSF HPLC method. 


\subsection{Effect of copper on the $10 \%$ w/w DSF-loaded PLGA nanoparticles}

$5 \mathrm{mg}$ of each $10 \% \mathrm{w} / \mathrm{w}$ disulfiram loaded PLGA nanoparticle formulation $(n=5)$ was placed into $1 \mathrm{ml}$ of PBS containing 10ul of copper and left for 72 hours. The PBS was centrifuged 10,000rpm for 5 minutes, the supernatant removed and the precipitated particles washed with $1 \mathrm{ml}$ of distilled water, centrifuged again and supernatant removed. The particles were dissolved in DCM and left overnight for the DCM to evaporate. The residue was dissolved in ethanol, filtered (pore size $0.22 \mu \mathrm{m}$, Millipore) and subsequently analysed using the stability indicating DSF HPLC method.

2.8. Determination of the serum half-life of DSF in the $10 \% \mathrm{~W} / \mathrm{W}$ DSF-loaded PLGA nanoparticles.

To determine the half-life of encapsulated DSF $5 \mathrm{mg}$ of each $10 \% \mathrm{w} / \mathrm{w}$ disulfiram loaded PLGA nanoparticle formulation $(n=5)$ was added into an eppendorf tube containing $300 \mu \mathrm{l}$ of horse serum with shaking at $37^{\circ} \mathrm{C}$. At set time intervals $(2,4,10$, 20, 30, 60 and 120 minutes) the eppendorf tubes were centrifuged at 10,000 rpm for 5 minutes, the supernatant removed and the precipitated particles washed with $1 \mathrm{ml}$ of distilled water, centrifuged again and supernatant removed. The particles were dissolved in DCM and left overnight for the DCM to evaporate. The residue was dissolved in ethanol, filtered (pore size $0.22 \mu \mathrm{m}$, Millipore) and subsequently analysed using the stability indicating DSF HPLC method.. The stability of the DSF in the supernatant was determined using the stability indicating DSF HPLC method. 


\subsection{DSF Stability Indicating HPLC Method}

HPLC analysis was performed on an Agilent 1200 series HPLC (Agilent, Santa Clara, CA) with a Phenomenex Luna C18 $4.6 \times 150 \mathrm{~mm} 2$ column with a 5-:M particle size (Phenomenex, Torrance, CA). The mobile phase comprises $80 \%$ HPLC grade methanol and 20\% HPLC grade water. The flow rate was $1.00 \mathrm{~mL} / \mathrm{min}$, whereas UV detection was performed at a wavelength of $275 \mathrm{~nm}$ with an injection volume of $10 \mu \mathrm{L}$.

2.10. The cytotoxicity of the $10 \% \mathrm{~W} / \mathrm{w}$ DSF-loaded PLGA nanoparticles against NSCLC cells

For in-vitro cytotoxicity analysis, the overnight cultured A549 lung cancer cells (5000/well in 96-well flat-bottomed microtitre plates) were constantly exposed to different concentrations of the $10 \%$ w/w DSF-loaded PLGA nanoparticles with the same concentration $(10 \mu \mathrm{m})$ of $\mathrm{CuCl} 2$ for $72 \mathrm{~h}$ and then subjected to a standard 3(4,5-Dimethylthiazol-2-yl)-2,5-Diphenyltetrazolium Bromide (MTT) assay as previously described [47]. Unformulated DSF was used as a control, the experiments repeated three times and the $50 \%$ inhibitory concentrations $\left(\mathrm{IC}_{50}\right)$ were calculated.

\subsection{Statistical Analysis}

Statistical analysis was performed using a one way analysis of variance (ANOVA) (GraphPad Prism version 5.02 for Windows, GraphPad Software, San Diego, CA). Post-hoc comparisons of the means were performed using Tukey's Honestly Significance Difference test. A significance level of $p<0.05$ was accepted to denote significance in all cases 


\section{Results and Discussion}

3.1. Surface morphology, particle size and zeta potential of the $10 \% \mathrm{~W} / \mathrm{w}$ DSF-loaded PLGA nanoparticles

The surface morphology of the $10 \% \mathrm{w} / \mathrm{w}$ DSF-loaded PLGA nanoparticles was analysed using SEM and a representative SEM image is presented in figure 1. The SEM image demonstrates that the nanoparticle formulations were spherical in shape, had a well-defined structure, a smooth surface with no pitting and did not aggregate together in small clusters. The smooth surface of the nanoparticles would indicate that there is no DSF on their surface, which suggests that the DSF was fully encapsulated and any excess washed away during preparation. Free DSF on the surface of the particle would mean less DSF was encapsulated and will influence DSF release resulting in an 'initial burst' [48]. Pitting can influence the durability and integratory of the nanoparticles [49] and tends to occur in acidic pH making the PLGA more porous and causing accelerated degradation, resulting in increased drug release, which could mean that a lower concentration of the drug will reach the target cell [50]. Aggregated nanoparticles are much more difficult to disperse making systemic administration difficult.

All of the nanoparticle formulations where in the nanoscale size range and their size ranged from 145.2 to $208.4 \mathrm{~nm}$ depending on the formulation and sonication time used (Table 2). The choice of PLGA had no significant effect on the size of the nanoparticles $(p<0.05)$ (Figure 2A), while increasing the PVA loading (Figure 2A) and sonication time (Figure 2B) significantly decreased the size of the nanoparticles $(p>0.05)$. Increasing the molecular weight of the PVA from 10 to $75 \mathrm{KDa}$ had no significant influence on their size $(p<0.05)$ (Figure 2B). However, when the 
molecular weight of the PVA was increased to $120 \mathrm{KDa}$ their size was significantly reduced $(p<0.05)$ (Figure $2 B)$. Increasing the PVA loading reduces particle size by increasing the stabilisation of the nanoparticles reducing aggregation, which in turn reduces the formation of larger particles [51]. Increasing the sonication time reduces the particle size by increasing shear stress, as a result of the longer sonication time, which increases droplet breakdown reducing the size of the particle [52]. Increasing the molecular weight of the PVA decreased the particle size because at a high molecular weight the PVA has less interaction with the aqueous phase thus further stabilizing the nanoparticles and reducing their size [51].

Zeta potential is a measure of the charge on the surface of a nanoparticle and can be used as an indicator of nanoparticle stability. When the surface charge is high there is a strong electrostatic repulsion between the nanoparticles, which results in reduced aggregation [53-54]. The minimum zeta potential required for stability is \pm 30 $\mathrm{mV}$ and all of the nanoparticles were positively charged with a zeta potential range of +35.2 to $+57.5 \mathrm{mV}$ (Table 2). Furthermore, zeta potential is an important feature in relation to the interaction of nanoparticles with cell membranes which is influenced by the surface charge of the nanoparticles [55]. The overall surface charge of cancer cells is normally negative [53] and positively charged nanoparticles strongly bind to the negatively charged cell membrane. Furthermore, positive nanoparticles have a much greater cytotoxicity compared to neutral nanoparticles due to being more efficient at penetrating and crossing the cell membrane of the cancer cell through endocytosis [55-57]. There was no correlation between zeta potential and the choice of PLGA, PVA loading, sonication time or PVA molecular weight (Table 2). 


\subsection{Encapsulation efficiency of the $10 \%$ w/w DSF-loaded PLGA nanoparticles}

The encapsulation efficiency of the nanoparticles ranged from 69 to $94 \%$ depending on the choice of formulation and sonication time (Figures 2C and D). The choice of PLGA polymer had no significant $(p>0.05)$ influence on the encapsulation efficiency of the nanoparticles (Figure $2 \mathrm{C}$ ). This is not surprising as all three polymers had the same ratio (50:50) of lactide to glycolide and similar molecular weights. The choice of PLGA can influence encapsulation efficiency but only when lactide to glycolide ratio or molecular weight varies. A PLGA with a high lactide to glycolide ratio or a low molecular weight will be highly soluble in the organic solvent, which can result in low encapsulation efficiencies [58]. As the PVA loading increased the encapsulation efficiency of the nanoparticles significantly decreased $(p<0.05)$ (Figure $2 C$ ). This is due to the size of the nanoparticles decreasing with an increase in PVA loading (Figure 2A). The smaller the size of the nanoparticles the less DSF they can encapsulate and thus the lower the encapsulation efficiency [59]. Furthermore, increasing the loading of PVA increases the concentration of residual PVA [60], which binds to the DSF limiting it's encapsulation in the PLGA [61]. Increasing the molecular weight of the PVA significantly decreased $(p<0.05)$ the encapsulation efficiency of the nanoparticles (Figure 2D), which again is due to the reduction in the size of the nanoparticles (Figure 2B), limiting the amount of DSF they can encapsulate. The longer the sonication time the lower the encapsulation efficiency of the nanoparticles (Figure 2D), which like PVA loading and molecular weight is due to a reduction in their size. However, it has also been demonstrated that increasing the sonication time can cause the PLGA polymer to prematurely degrade increasing its permeability, causing the drug to diffuse out during washing [62]. 


\subsection{In vitro DSF release from the $10 \% \mathrm{~W} / \mathrm{w}$ DSF-loaded PLGA nanoparticles}

In vitro release of DSF from the PLGA nanoparticles over 7 days is presented in figure 3. All of the nanoparticle formulations had diffusion controlled release profiles typically associated with PLGA nanoparticles (Figure 3). The choice of PLGA had a significant $(p<0.05)$ influence on the release of DSF from the nanoparticles (Figure $3 \mathrm{~A})$, which is due to the degradation rate of the PLGA. DLG 1E has a degradation rate of days, while DLG $4 \mathrm{~A}$ and $4 \mathrm{E}$ have degradation rates of weeks and months respectively. We have previous demonstrated that the choice of PLGA polymer can influence the release of DSF from PLGA based devices [31-32]. Increasing the loading of PVA in the formulation significantly $(p<0.05)$ increased the release rate of DSF from the nanoparticles (Figure 3B). This is due to a decrease in the size of the nanoparticles with an increase in PVA loading (Figure 2A), which results in an increase in their overall surface area. This increase in surface area resulted in a significant $(p<0.05)$ increase in the day 1 burst for the nanoparticles containing $8 \%$ w/w PVA when compared to the nanoparticles containing either a 2 or $4 \% \mathrm{w} / \mathrm{w}$ PVA loading (Figure 3B). The was no significant $(p>0.05)$ difference in the release of DSF from day 1 to day 5 for the nanoparticles containing PVA with a molecular weight of 10 and $75 \mathrm{KDa}$ (Figure $3 \mathrm{C}$ ). This is due to these nanoparticles being of similar size (Figure 2B). However, when the molecular weight of the PVA was increased to $120 \mathrm{KDa}$ the release of DSF significantly $(p<0.05)$ increased (Figure 3C) due to the reduced particle size of the nanoparticles (Figure 2B), which resulted in an increase in their overall surface area. This is demonstrated by a dramatic increase in their day 1 burst when compared to the nanoparticles containing PVA with a molecular weight of 10 and $75 \mathrm{KDa}$ (Figure 3C). Increasing sonication time significantly $(p<0.05)$ increased the release of DSF from the nanoparticles (Figure 
3D) again due to a decrease in their size (Figure 2B), which results in an increase in their overall surface area. There is a substantial increase in the day 1 burst of the nanoparticles sonicated for 9 minutes when compared to those sonicated for 3 and 6 minutes (Figure 3D). The reduced size of the nanoparticles and increase in overall surface area is a contributing factor. However, another contributing factor is the increased permeability of the nanoparticles due to premature degradation of the PLGA associated with the increased sonication time [62].

3.4. The ability of the $10 \% \mathrm{~W} / \mathrm{W}$ DSF-loaded PLGA nanoparticles to protect DSF from the premature chelating of copper

The anticancer effect of DSF has been shown to be Cu dependent [37-38] and due to cancer cells having increased copper levels compared to normal cells [63] DSF demonstrates selectivity towards cancer cells compared to normal cells. We have recently demonstrated that DSF has two mechanisms of action for killing cancer cells: 1) Instant cell death caused by the generation of ROS as a result of the DSF/Cu reaction and 2) Delayed cell death caused by the cytotoxic nature of the DSF/Cu complex [64]. However, in order for these mechanisms to be effective the $\mathrm{DSF} / \mathrm{Cu}$ reaction must take place within the cancer cell. Therefore, it is imperative that the nanoparticles protect the DSF from reacting with $\mathrm{Cu}$ outside of the cancer cell.

Figure 4 demonstrates that the encapsulation of DSF into PLGA nanoparticles provides protection from copper in comparison with an unformulated DSF control. However, the level of protection does depend on the formulation and sonication time used. The choice of PLGA has no significant $(p>0.05)$ influence on the level of DSF protection that the nanoparticles provide (Figure 4A), which is due to the size of the 
nanoparticles being similar (Figure 2A). We believe that the level of DSF protection is based on a number of factors: 1) the size of the nanoparticles, 2) the overall surface area of the nanoparticle formulation and 3) the permeability of the nanoparticles. Therefore, the bigger the nanoparticle the greater the protection they provide due to the $\mathrm{Cu}$ having further to diffuse to have access to the DSF and the overall surface area being lower. This is demonstrated by the fact that as the PVA loading, PVA molecular weight and sonication time are increased the level of protection from $\mathrm{Cu}$ each nanoparticle formulation provides is decreased (Figure 4A and $4 \mathrm{~B}$ ). This correlates with a decrease in particle size (Figure 2A and $2 \mathrm{~B}$ ) and an increase in the surface area of the nanoparticle formulation. Furthermore, the level of protection provided by the nanoparticle formulation that had a 9 minute sonication time is significantly lower than all of the other nanoparticle formulations (Figure $4 \mathrm{~A}$ and 4B). This is due to not only the decreased particle size and increased surface area, but also to the increased permeability of the PLGA caused by premature degradation as a result of the increased sonication time.

3.5. The ability of the $10 \% \mathrm{~W} / \mathrm{W}$ DSF-loaded PLGA nanoparticles to protect DSF from degradation in serum

In order for DSF to have an anticancer effect it must first accumulate in the cancer cells in its unaltered form allowing it to chelate with copper. However, DSF has a very short half-life (4 minutes) in the bloodstream, which is limiting its anticancer application in the clinic. Therefore, a drug delivery system which protects DSF from metabolism in the blood stream would expedite its translation into the clinic as a treatment for NSCLC. We recently demonstrated that PLGA nanoparticles can extend the half-life of DSF in blood from under 2 minutes to 7 hours [46]. To further develop our DSF-loaded PLGA nanoparticles it is imperative that we understand how 
the choice of formulation and sonication time influences the ability of the PLGA nanoparticles to protect the DSF from degradation in the bloodstream.

Figure 5 demonstrates that all of the DSF-loaded PLGA nanoparticle formulations significantly $(p<0.05)$ increased the half-life of DSF in serum in comparison to an unformulated DSF control. The choice of PLGA had no significant $(p>0.05)$ influence on increasing the half-life of DSF in serum (Figure 5A). All three PLGA polymers increased its half-life from approximately 3 minutes to 60 minutes, with approximately $40 \%$ of the DSF remaining after 120 minutes. As with the protection of DSF form $\mathrm{Cu}$, its level of protection in serum is also due to the size of the nanoparticles, their overall surface area and their permeability. The PLGA coating protects the DSF from the serum for longer, thus increasing the half-life. However, the fact that the choice of PLGA has no influence on the size of the nanoparticles (Figure $2 \mathrm{~A}$ ) and thus no influence on the overall surface area the level of protection is similar for each PLGA. Furthermore, the PLGAs used had the same ratio of lactide to glycolide, similar molecular weights and the same sonication time was used to produce the nanoparticles. Thus, the level of permeability of the nanoparticles would be similar, resulting in the level of protection of DSF being similar.

The PVA loading and molecular weight had a significant influence $(p<0.05)$ on the increase in DSF half-life provided by the nanoparticles (Figure $5 \mathrm{~B}$ and $5 \mathrm{C}$ ). The 2, 4 and $8 \% \mathrm{w} / \mathrm{w}$ PVA loaded nanoparticles increased the half-life of DSF in serum from approximately 3 minutes to 120, 90 and 30 minutes respectively (Figure 5B). The 10, 75 and 120 KDa PVA nanoparticles increased the half-life of DSF in serum from 3 minutes to 90,55 and 22 minutes respectively (Figure $5 \mathrm{C}$ ). As mentioned previously the PLGA coating protects the DSF from the serum for longer, thus 
increasing the half-life. The trend in the level of protection is associated with the size of the nanoparticles and their overall surface area. Increasing both the PVA loading and molecular weight decreases the size of the nanoparticles (Figure 2A and 2B), which means that the serum has less distance to diffuse into the nanoparticle before it comes in contact with the DSF causing it to degrade. Furthermore, the smaller the nanoparticles the greater their overall surface area, which increases the diffusion of the serum into them, decreasing the length of time it takes for it to access the DSF causing it to degrade.

Increasing the sonication time significantly $(p<0.05)$ decreased the half-life of DS in the nanoparticles (Figure 5D). At 3, 6 and 9 minute sonication time the nanoparticles increased the half-life of DSF in serum from approximately 3 minutes to 90,20 and 10 minutes respectively (Figure 5D). As mentioned previously the decrease in DSF half-life is due to the decrease in the size of the nanoparticles and increase in their overall surface area. However, the decrease in half-life at 6 minutes and 9 minutes was significantly greater than what would be expected based on the decrease in particle size and thus another factor must be coming into play. We believe this factor to be the permeability of the PLGA. It has been demonstrated that increasing the sonication time can cause the PLGA polymer to prematurely degrade increasing its permeability [62], increasing the diffusion rate of the serum into the nanoparticle decreasing the length of time it takes for it to access the DSF causing it to degrade.

3.6. The cytotoxicity of the $10 \% \mathrm{w} / \mathrm{w}$ DSF-loaded PLGA nanoparticles against NSCLC cells

The cytotoxicity of the 10\% w/w DSF-loaded PLGA nanoparticles against NSCLC is presented in figure 6 . The figure demonstrates that all of the nanoparticle 
formulations are cytotoxic against NSCLC. However, their $\mathrm{IC}_{50}$ is significantly $(\mathrm{p}<$ 0.05) greater than that of free DSF $(7.6 n M)$. The choice of PLGA had no significant $(p>0.05)$ effect on the cytotoxicity of the nanoparticles with DLG 1E, 4A and 4E having an $\mathrm{IC}_{50}$ values of $14.6,16.1$ and $16.1 \mathrm{nM}$ respectively (Figure $6 \mathrm{~A}$ ). An increase in PVA loading increased the cytotoxicity of the nanoparticle formulations, with the 2, 4 and $8 \% \mathrm{w} / \mathrm{w}$ loadings having $\mathrm{IC}_{50}$ values of $32.3,16.2$ and 13.4 respectively (Figure 6B). Increasing the molecular weight of the PVA also increased the cytotoxicity of the nanoparticle formulations (Figure 6C). The 10, 75 and 120 KDa PVA nanoparticles had $I_{50}$ values of $25.8,16.2$ and 11.5 respectively. An increase in sonication time increased the cytotoxicity of the nanoparticle formulations, with the 3,6 and 9 minute sonication times having $I_{50}$ values of 16.2 , 10.2 and 9.8 respectively (Figure 6D).

This trend in cytotoxicity is predominately controlled by the particle size of the nanoparticles, which is demonstrated by a linear relationship between particle size and $\mathrm{IC}_{50}$ value (Figure $6 \mathrm{E}$ ). However, we believe that the permeability of the nanoparticles is also playing a part, particularly for those with a 6 and 9 minute sonication time. The nanoparticles with a 6 minute sonication time had a similar particle size $(152.7 \mathrm{~nm})$ to the $8 \% \mathrm{w} / \mathrm{w}$ PVA nanoparticles $(150.6)$ but had a significantly $(\mathrm{p}<0.05)$ lower $\mathrm{IC}_{50}$ value, 10.2 compared to $13.4 \mathrm{nM}$, making them more cytotoxic. The same trend occurs with those nanoparticles sonicated for 9 minutes. They had a particle size of $145.2 \mathrm{~nm}$, while the nanoparticles containing PVA with a molecular weight of $120 \mathrm{KDa}$ had a particle size of $145.9 \mathrm{~nm}$. However, the nanoparticles sonicated for 9 minutes had an $\mathrm{IC}_{50}$ value of 9.8 , while those containing PVA with a molecular weight of $120 \mathrm{KDa}$ had an $\mathrm{IC}_{50}$ value of $11.5 \mathrm{nM}$. We believe that the extra 3 and 6 minutes of sonication caused the PLGA to 
prematurely degrade, increasing their permeability, which increased the diffusion of DSF out the nanoparticles resulting in a greater concentration of DSF inside the NSCLC cells increasing cell death. This is further demonstrated by the fact that the nanoparticles sonicated for 6 and 9 minutes provided the highest release rate of DSF (Figure 3). 


\section{Conclusion}

This paper investigates the influence of PLGA type, PVA loading and molecular weight as well as sonication time on the morphology, particle size, zeta potential, encapsulation efficiency, DSF release, protection from copper, protection from degradation in serum and cytotoxicity of $10 \% \mathrm{w} / \mathrm{w}$ DSF-loaded PLGA nanoparticles. The choice of PLGA polymer had no significant influence on the any of the nanoparticle characteristics except for DSF release, which was due to the varying degradation times of the PLGAs. Increasing both the PVA loading and molecular weight decreased the size of the nanoparticles, which in turn increased the overall surface area of the nanoparticle formulation. The decrease in particle size and increase in overall surface area reduced the ability of the nanoparticles to protect the DSF from reacting with $\mathrm{Cu}$ and degradation in serum, while increasing their DSF release rate and cytotoxicity. Increasing the sonication time also decreased the size of the nanoparticles and thus increased their overall surface area. Like increasing PVA loading and molecular weight this resulted in a decrease in the protection of DSF from reacting with $\mathrm{Cu}$ and degradation in serum, while increasing their DSF release and cytotoxicity. Additionally, increasing the sonication time resulted in the premature degradation of the PLGA, which increased the permeability of the nanoparticles. This increase in permeability further decreased their ability to protect DSF from reacting with $\mathrm{Cu}$ and degradation in serum, while further increasing their DSF release rate and cytotoxicity. We have recently demonstrated that a low dose of DSF-loaded PLGA nanoparticles supplemented with oral $\mathrm{Cu}$ inhibited tumour growth and reduced metastasis in a xenograft mouse lung cancer model. This paper demonstrates the influence of the various formulation parameters and sonication time on the characteristics and cytotoxicity of the DSF-loaded PLGA nanoparticles, 
which will allow us to produce the most stable and cytotoxic nanoparticle formulation. Furthermore, this research will inform the scale-up and full scale manufacture of a DSF-loaded nanoparticle formulation expediting its translation from the lab into the clinic. 


\section{Figure Captions}

Figure 1: Representative SEM image of the $10 \% \mathrm{w} / \mathrm{w}$ DSF-loaded PLGA nanoparticles

Figure 2: Particle size (A and $\mathbf{B})$ and encapsulation efficiency (C and $\mathbf{D})$ of the $10 \%$ w/w DSF-loaded PLGA nanoparticles

Figure 3: In vitro drug release for the $10 \% \mathrm{w} / \mathrm{w}$ DSF-loaded PLGA nanoparticles prepared using different PLGA polymers (A), PVA loadings (B), PVA molecular weights (C) and sonication times (D).

Figure 4: The influence of PLGA polymer and PVA loading (A) and well as PVA molecular weight and sonication time (B) on the ability of the 10\% w/w DSF-loaded nanoparticles to protect DSF from reacting with $\mathrm{Cu}$

Figure 5: The influence of PLGA polymer (A), PVA loading (B), PVA molecular weight (C) and sonication time (D) on the ability of the $10 \% \mathrm{w} / \mathrm{w}$ DSF-loaded nanoparticles to protect DSF from degradation in serum.

Figure 6: The influence of PLGA polymer (A), PVA loading (B), PVA molecular weight (C) and sonication time (D) on the cytotoxicity of the $10 \% \mathrm{w} / \mathrm{w}$ DSF-loaded nanoparticles in comparison to a DSF control. The relationship between particle size and the $\mathrm{IC}_{50}$ value of the $10 \% \mathrm{w} / \mathrm{w}$ DSF-loaded nanoparticles $(\mathbf{E})$.

\section{Table Captions}

Table 1: The formulation variables and sonication times of the $10 \% \mathrm{w} / \mathrm{w}$ DSF-loaded PLGA nanopartilces. 
Table 2: Particle size and Zeta potentials for the $10 \% \mathrm{w} / \mathrm{w}$ DSF-loaded nanoparticles. 


\section{References}

1. www.cancer.org/cancer/lungcancer-non-smallcell/detailedguide/non-smallcell-lung-cancer-key-statistics

2. KA. Houston, SJ. Henley, J. Li, MC. White, TB. Richards, Patterns in lung cancer incidence rates and trends by histologic type in the United States, 2004-2009. Lung Cancer. (2014) 86:22-28.

3. K. Ohgino, K. Soejima, H. Yasuda, Y. Hayashi, J. Hamamoto, K. Naoki, D. Arai, K. Ishioka, T. Sato, H. Terai, S. Ikemura, S. Yoda, T. Tani, A. Kuroda, T. Betsuyaku, Expression of fibroblast growth factor 9 is associated with poor prognosis in patients with resected non-small cell lung cancer. Lung Cancer. (2014) 83:90-96.

4. S. Yoda, K. Soejima, J. Hamamoto, H. Yasuda, S. Nakayama, R. Satomi, H. Terai, S. Ikemura, T. Sato, K. Naoki, T. Betsuyaku, Claudin-1 is a novel target of miR-375 in non-small-cell lung cancer. Lung Cancer. (2014) 85:366372.

5. N. Zhao, X. Zhang, H. Yan, J. Yang, Y. Wu, Y, Efficacy of epidermal growth factor receptor inhibitors versus chemotherapy as second-line treatment in advanced non-small-cell lung cancer with wild-type EGFR: A meta-analysis of randomized controlled clinical trials. Lung Cancer. (2014) 85:66-73.

6. H. Chen, X. Zhou, Y. Gao, B. Zheng, F. Tang, J. Huang, Recent progress in development of new sonosensitizers for sonodynamic cancer therapy. Drug Discov. Today. (2014) 19:502-509.

7. I. Kim, HJ. Byeon, TH. Kim, ES. Lee, KT. Oh, BS. Shin, KC. Lee, YS. Youn, Doxorubicin-loaded highly porous large PLGA microparticles as a sustained- 
release inhalation system for the treatment of metastatic lung cancer. Biomaterials. (2012) 33:5574-5583.

8. I. Kim, HJ. Byeon, TH. Kim, ES. Lee, KT. Oh, BS. Shin, KC. Lee, KC. and Youn, Doxorubicin-loaded porous PLGA microparticles with surface attached TRAIL for the inhalation treatment of metastatic lung cancer. Biomaterials. (2013) 34:6444-6453.

9. CM. Hu, LF. Zhang, Therapeutic nanoparticles to combat cancer drug resistance. Curr Drug Metab (2009) 10:836-841.

10. KJ. Cho, X. Wang, SM. Nie, Z. Chen, DM. Shin, Therapeutic nanoparticles for drug delivery in cancer. Clin. Cancer Res. (2008) 14:1310-1316.

11. H. Maeda, J. Wu, T. Sawa, Y. Matsumara, K. Hori, Tumor vascular permeability and the EPR effect in macromolecular therapeutics: a review. J. Con. Rel. (200) 65:271-284.

12. H. Maeda, J. Fang, T. Inutsuka, Y. Kitamoto, Vascular permeability enhancement in solid tumor: various factors, mechanisms involved and its implications. Int. Immunopharmacol. (2003) 3:319-328.

13. I. Brigger, C. Dubernet, P. Couvreur, Nanoparticles in cancer therapy and diagnosis. Adv. Drug Deliv. Rev. (2002) 54:631-651.

14.U. Gaur, SK. Sahoo, TK. De, PC. Ghosh, A. Maitra, PK. Ghosh, Biodistribution of fluoresceinated dextran using novel nanoparticles evading reticuloendothelial system. Int. J. Pharm. (2000) 202:1-10.

15. G. Storm, SO. Belliot, T. Daemen, D. Lasic, Surface modification of nanoparticles to oppose uptake by the mononuclear phagocyte system. Adv. Drug Deliv. Rev. (1995) 17:31-48. 
16. YW. Wang, H. Sato, I. Dachi, I. Horikoshi, Preparation and characterization of poly(lactic-co-glycolic acid) microspheres for targeted delivery of novel anticancer agent taxol. Chem. Pharm. Bull. (1996) 44:1935-1940.

17. F. Si-Shen, H. Guofeng, Effect of emulsifiers on the controlled release of paclitaxel (Taxol) from nanospheres of biodegradable polymer. J. Con. Rel. (2001) 71:53-69.

18. C. Chittasupho, K. Lirdprapamongkol, P. Kewsuwan, N. Sarisuta, Targeted delivery of doxorubicin to A549 lung cancer cells by CXCR4 antagonist conjugated PLGA nanoparticles. Eur. J. Pharm. Biopharm. (2014) 88:529538.

19.S. Vinogradov , X. Wei, Cancer stem cells and drug resistance: the potential of nanomedicine. Nanomedicine (2012) 7:597-615.

20.J. Codony-Servat, A. Verlicchi, R. Rosell, Cancer stem cells in small cell lung cancer. Transl. Lung Cancer Res. (2016) 5:16-25.

21. NC. Yip, IS. Fombon, P. Liu, S. Brown, V. Kannappan, A.L. Armesilla, B. Xu, J. Cassidy, JL. Darling, W. Wang, Disulfiram modulated ROS-MAPK and NFkB pathways and targeted breast cancer cells with cancer stem cell-like properties. Br. J. Cancer. (2011) 104:1564-1574

22. P. Liu, S. Brown, T. Goktug, P. Channathodiyil, V. Kannappan, JP. Hugnot, PO. Guichet, X. Bian, AL. Armesilla, JL. Darling, W. Wang, Cytotoxic effect of disulfiram/copper on human glioblastoma cell lines and ALDH-positive cancerstem-like cells. Br. J. Cancer. (2012) 107:1488-1497

23. D. Chen, QC. Cui, H. Yang, QP. Dou, Disulfiram, a Clinically Used AntiAlcoholism Drug and Copper-Binding Agent, Induces Apoptotic Cell Death in 
Breast Cancer Cultures and Xenografts via Inhibition of the Proteasome Activity. Cancer Res. (2006) 66:10425-10433

24. K. Iljin, K. Ketola, P. Vainio, P. Halonen, P. Kohonen, V. Fey, RC. Grafström, M. Perälä, O. Kallioniemi, High-Throughput Cell-Based Screening of 4910 Known Drugs and Drug-like Small Molecules Identifies Disulfiram as an Inhibitor of Prostate Cancer Cell Growth. Clin. Cancer Res. (2009) 15:60706078

25. K. Ketola, O. Kallioniemi, K. Iljin, Chemical biology drug sensitivity screen identifies sunitinib as synergistic agent with disulfiram in prostate cancer cells, PloS one. (2012) 7:e51470

26. SS. Brar, C. Grigg, KS. Wilson, WD. Holder, D. Dreau, C. Austin, M. Foster, AJ. Ghio, AR. Whorton, GW. Stowel, LB. Whittall, RR. Whittle, DP. White, TP. Kennedy, Disulfiram inhibits activating transcription factor/cyclic AMPresponsive element binding protein and human melanoma growth in a metaldependent manner in vitro, in mice and in a patient with metastatic disease, Mol. Cancer Ther. (2004) 3:1049-1060

27. P. Dufour, JM. Lang, C. Giron, B. Duclos, P. Haehnel, D. Jaeck, JM. Jung, F. Oberling, Sodium ditiocarb as adjuvant immunotherapy for high risk breast cancer: A randomized study. Biotherapy. (1993) 6:9-12

28. S. Verma, DJ. Stewart, JA. Maroun, RC. Nair, A Randomized Phase II Study of Cisplatin Alone Versus Cisplatin Plus Disulfiram. Am. J. Clin. Oncol. (1990) 13:119-124

29. J. Triscott, C. Lee, K. Hu, A. Fotovati, R. Berns, M. Pambid, M. Luk, RE. Kast, E. Kong, E. Toyota, S. Yip, B. Toyota, SE. Dunn, Disulfiram, a drug widely 
used to control alcoholism, suppresses the self-renewal of glioblastoma and over-rides resistance to temozolomide. Oncotarget. (2012) 3:1112-1123.

30. P. Hothi, TJ. Martins, L. Chen, L. Deleyrolle, JG. Yoon, B. Reynolds, G. Foltz, High throughput chemical screens identify disulfiram as an inhibitor of human glioblastoma stem cells. Oncotarget. (2012) 3:1124-1136.

31. I. Zembko, I. Ahmed, A. Farooq, J. Dail, P. Tawari, W. Wang, C. Mcconville, Development of Disulfiram-Loaded Poly(Lactic-co-Glycolic Acid) Wafers for the Localised Treatment of Glioblastoma Multiforme: A Comparison of Manufacturing Techniques. J. Pharm. Sci. (2014) 104:1076-1086.

32. C. McConville, P. Tawari, W. Wang, Hot melt extruded and injection moulded disulfiram-loaded PLGA millirods for the treatment of glioblastoma multiforme via stereotactic injection. Int. J. Pharm. (2015) 494:73-82.

33. CS. Baffoe, N. Nguyen, P. Boyd, W. Wang, M. Morris, C. McConville, Disulfiram-loaded immediate and extended release vaginal tablets for the localised treatment of cervical cancer. J. Pharm. Pharmacol. (2015) 67:18998.

34. P. Boyd, I. Major I, W, Wang, C. McConville, Development of disulfiramloaded vaginal rings for the localised treatment of cervical cancer. Eur. J. Pharm. Biopharm. (2014) 88:945-53.

35. P. Liu, IS. Kumar, S. Brown, V. Kannappan, PE. Tawari, JZ. Tang, W. Jiang, AL. Armesilla, JL. Darling, W. Wang, Disulfiram targets cancer stem-like cells and reverses resistance and cross-resistance in acquired paclitaxel-resistant triple-negative breast cancer cells. Br. J. Cancer. (2013) 109:1876-1885.

36. P. Liu, Z. Wang, S. Brown, V. Kannappan, PE. Tawari, J. Jiang, JM. Irache, JZ. Tang, AL. Armesilla, JL. Darling, X. Tang W. Wang, Liposome 
encapsulated Disulfiram inhibits NFKB pathway and targets breast cancer stem cells in vitro and in vivo. Oncotarget (2014) 5:7471-7485.

37. D. Cen, RI. Gonzalez, JA. Buckmeier, RS. Kahlon, NB. Tohidian, FL. Meyskens, Disulfiram induces apoptosis in human melanoma cells: a redoxrelated process. Mol. Cancer Ther. (2002) 1:197-204.

38. D. Cen, D. Brayton, B. Shahandeh, FL. Meyskens, PJ. Farmer, Disulfiram facilitates intracellular $\mathrm{Cu}$ uptake and induces apoptosis in human melanoma cells. J. Med. Chem. (2004) 47:6914-6920.

39. DG. Barceloux, Copper, J. Toxicol. Clin. Toxicol. (1999) 37:217-230.

40. H. Nechushtan, Y. Hamamreh, S. Nidal, M. Gotfried, A. Baron, YI. Shalev, B. Nisman, T. Peretz, N. Peylan-Ramu, A phase llb trial assessing the addition of disulfiram to chemotherapy for the treatment of metastatic non-small cell lung cancer. Oncologist. (2015) 20:366-367.

41. MJ. Burkitt, HS. Bishop, L. Milne, SY. Tsang, GJ. Provan, CS. Nobel, S. Orrenius, AF. Slater, Dithiocarbamate toxicity toward thymocytes involves their copper-catalyzed conversion to thiuram disulfides, which oxidize glutathione in a redox cycle without the release of reactive oxygen species, Arch. Biochem. Biophys. (1998) 353:73-84.

42. Cl. Nobel, M. Kimland, B. Lind, S. Orrenius, AF. Slater, Dithiocarbamates induce apoptosis in thymocytes by raising the intracellular level of redoxactive copper. J. Biol. Chem. (1995) 270:26202-26208.

43. H. Nakano, A. Nakajima, S. Sakon-Komazawa, JH. Piao, X. Xue, K. Okumura, Reactive oxygen species mediate crosstalk between NF-kappaB and JNK. Cell Death Differ. (2006) 13:730-737. 
44. NC. Yip, IS. Fombon, P. Liu, S. Brown, V. Kannappan, AL. Armesilla, B. Xu, J. Cassidy, JL. Darling, W. Wang, Disulfiram modulated ROS-MAPK and NFkB pathways and targeted breast cancer cells with cancer stem cell-like properties. Br. J. Cancer. (2011) 104:1564-1574.

45. V. Kannappan, K. Butcher, Z. Wang, C. McConville, W. Wang, Antialcoholism drug gives lung cancer patients new hope. Poster presentation at the British Lung Foundation Alumni Conference, $7^{\text {th }}$ March 2016.

46. Z. Wang, J. Tan, C. McConville, V. Kannappan, P. Tawari, J. Ding, J. Brown, A. Armesilla, J. Irache, QB. Mei, T. Tan, W. Jiang, Y. Liu, X. Bian, W. Wang, Poly lactic-co-glycolic acid controlled delivery of Disulfiram to target liver cancer stem-like cells. Nanomedicine. (2016) Accepted for publication, In press.

47. JA. Plumb, R. Milroy, SB. Kaye, Effects of the $\mathrm{pH}$ dependence of 3-(4,5dimethylthiazol-2-yl)-2,5-diphenyl-tetrazolium bromide-formazan absorption on chemosensitivity determined by a novel tetrazolium-based assay. Cancer Res. (1989) 49:4435-4440.

48. HK. Makadia, SJ. Siegel, Poly Lactic-co-Glycolic Acid (PLGA) as Biodegradable Controlled Drug Delivery. Polymers (Basel) (2011) 3:13771397.

49. Z. Chen, F. Bobaru, Peridynamic modelling of pitting corrosion damage. J. Mech. Phys. Solids. (2015) 78:352-381

50.BS. Zolnik, DJ. Burgess,(2007) Effect of acidic pH on PLGA microsphere degradation and release. J. Con. Rel. (2015) 122:338-344. 
51. RM. Mainardes, RC. Evangelista, PLGA nanoparticles containing praziquantel: effect of formulation variables on size distribution. Int. J. Pharm. (2005) 290:137-144.

52. A. Budhiana, SJ. Siegelb, KI. Winey, Haloperidol-loaded PLGA nanoparticles: Systematic study of particle size and drug content. Int. J. Pharm. (2007) 336:367-375.

53. S. Honary, F. Zahir, Effect of Zeta Potential on the Properties of Nano-Drug Delivery Systems - A Review (Part 2). Trop. J. Pharm. Res. (2013) 12:265273.

54. ML. Hans, AM. Lowman, Biodegradable nanoparticles for drug delivery and targeting. Curr. Opin. Solid State Mater. Sci. (2002) 6:319-327.

55. S. Jose, S. Sowmya, TA. Cinu, NA. Aleykutty, S. Thomas, EB. Souto, Surface modified PLGA nanoparticles for brain targeting of Bacoside-A. Eur. J. Pharm. Sci. (2014) 63:29-35.

56. L. Chen, JM. Mccrate, JC. Lee, H. Li, The role of surface charge on the uptake and biocompatibility of hydroxyapatite nanoparticles with osteoblast cells. Nanotechnology. (2011) 22:1-10.

57. A. Verma, F. Stellacci, Effect of Surface Properties on Nanoparticle-Cell Interactions. Small. (2010) 6:12-21.

58. NV. Jyothi, PM. Prasanna, SN. Sakarkar, KS. Prabha, PS. Ramaiah, GY. Srawan, Microencapsulation techniques, factors influencing encapsulation efficiency. J. Microencapsul. (2010) 27:187-97.

59. M. Halayqa, U. Domańska, PLGA Biodegradable Nanoparticles Containing Perphenazine or Chlorpromazine Hydrochloride: Effect of Formulation and Release. Int. J. Mol. Sci. (2014) 15:23909-23923. 
60. SK. Sahooa, J. Panyama, S. Prabhaa, V. Labhasetwara, Residual polyvinyl alcohol associated with poly (d,I-lactide-co-glycolide) nanoparticles affects their physical properties and cellular uptake. J. Con. Rel. (2002) 82:105-114.

61.S. Fenga, G. Huangb (2001) Effects of emulsifiers on the controlled release of paclitaxel $($ Taxolß $)$ from nanospheres of biodegradable polymers. J. Con. Rel. (2001) 7153-69.

62. PB. O'Donnell, JW. McGinity, Preparation of microspheres by the solvent evaporation technique. Adv. Drug Deliv. Rev. (1997) 28:25-42

63. A. Gupte, RJ. Mumper, Elevated copper and oxidative stress in cancer cells as a target for cancer treatment. Cancer Treat. Rev. (2009) 35:32-46.

64.P. Tawari, Z. Wang, M. Najlah, C. Tsang, V. Kannappan, P. Liu, C. McConville, B. He, A. Armesilla, W. Wang, The cytotoxic mechanisms of disulfiram and copper(II) in cancer cells. Toxicol. Res. (2015) 4:1439-1442. 

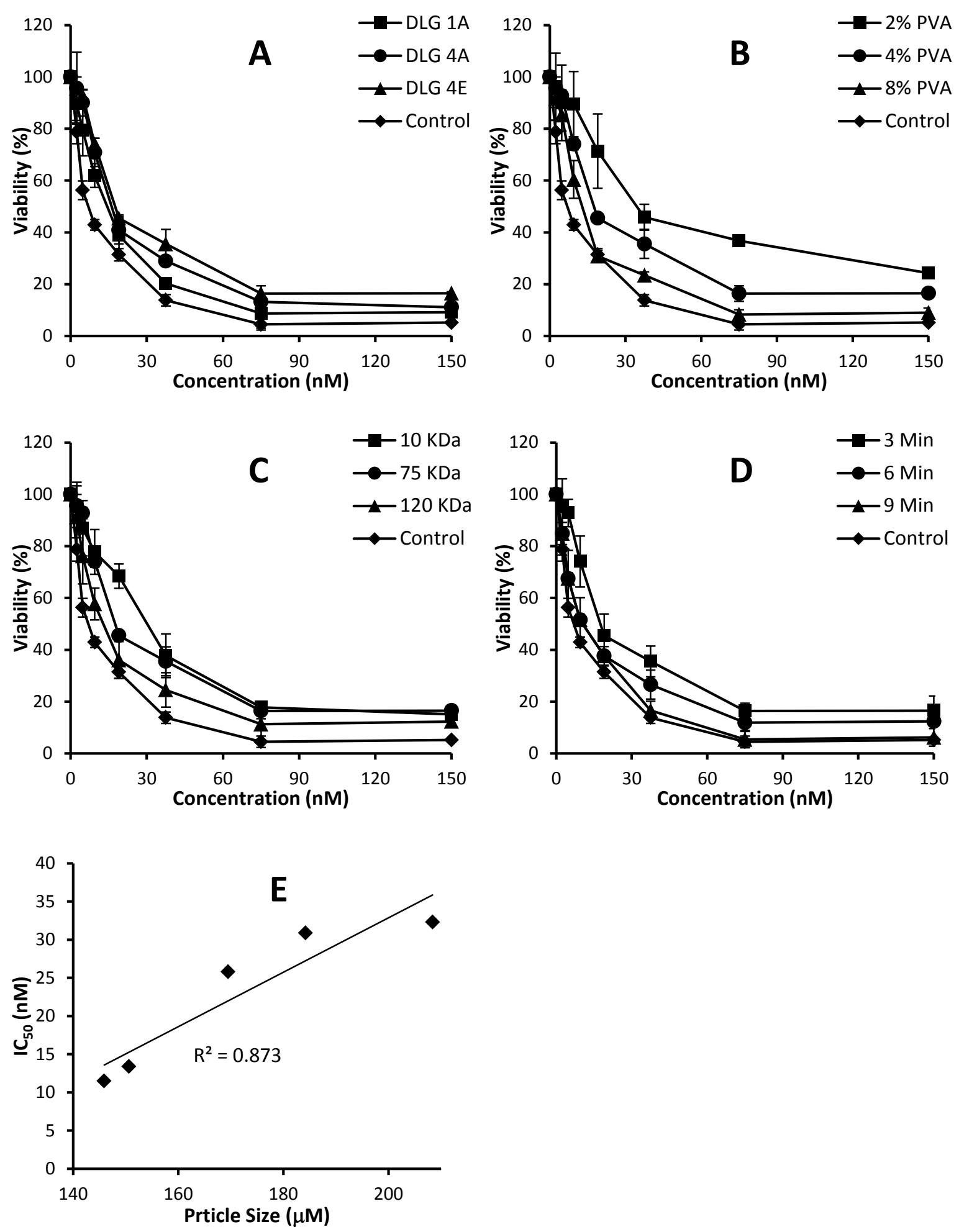
Figure 5
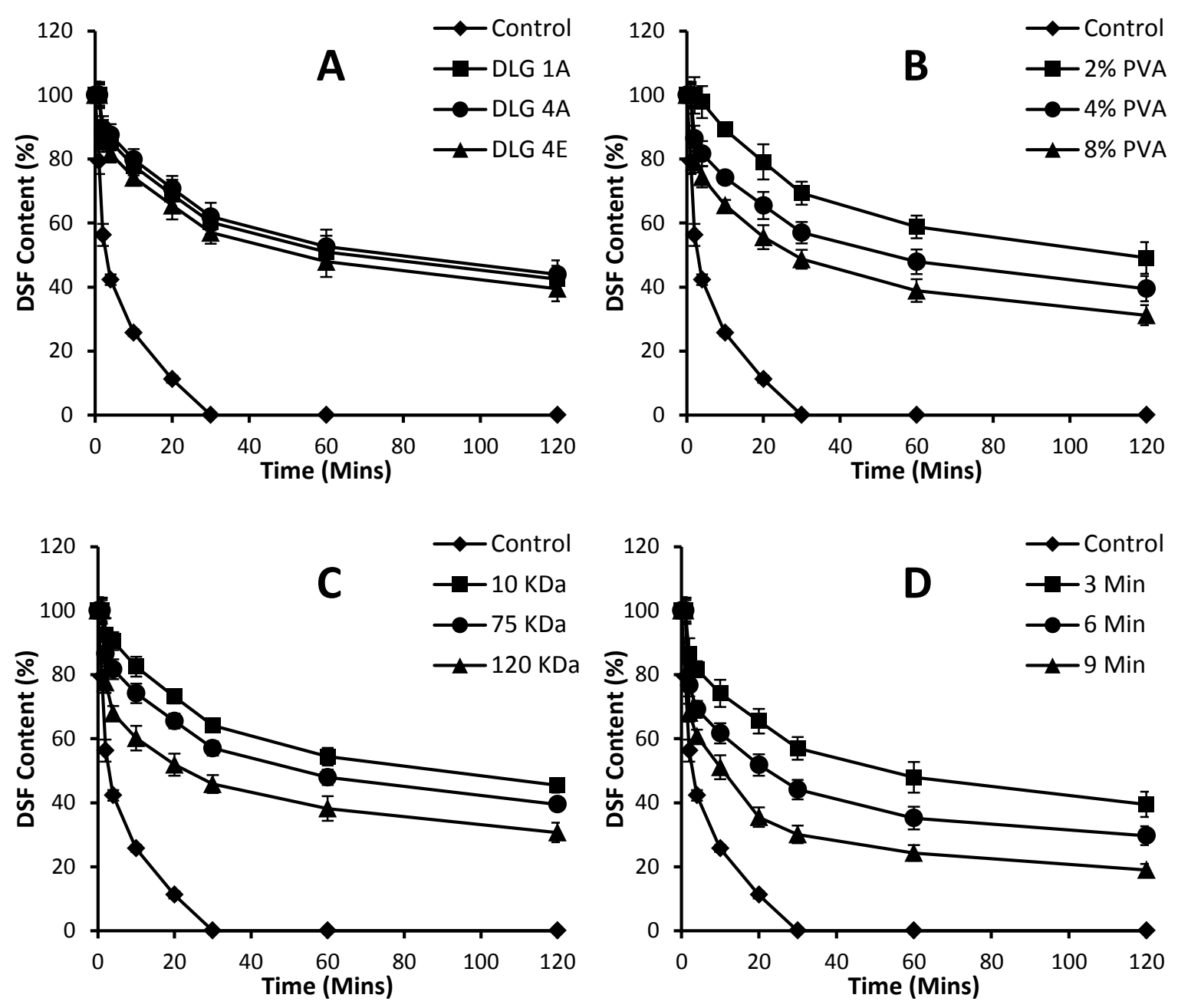
Figure 4
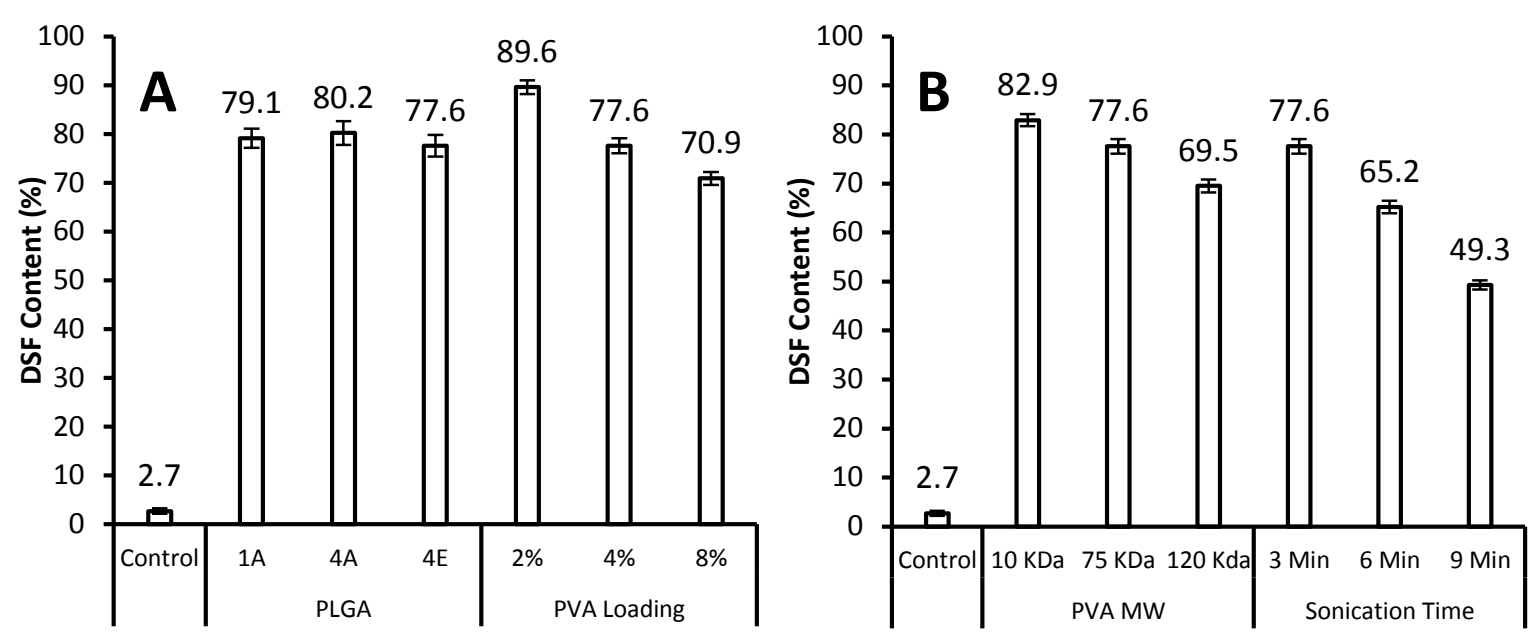

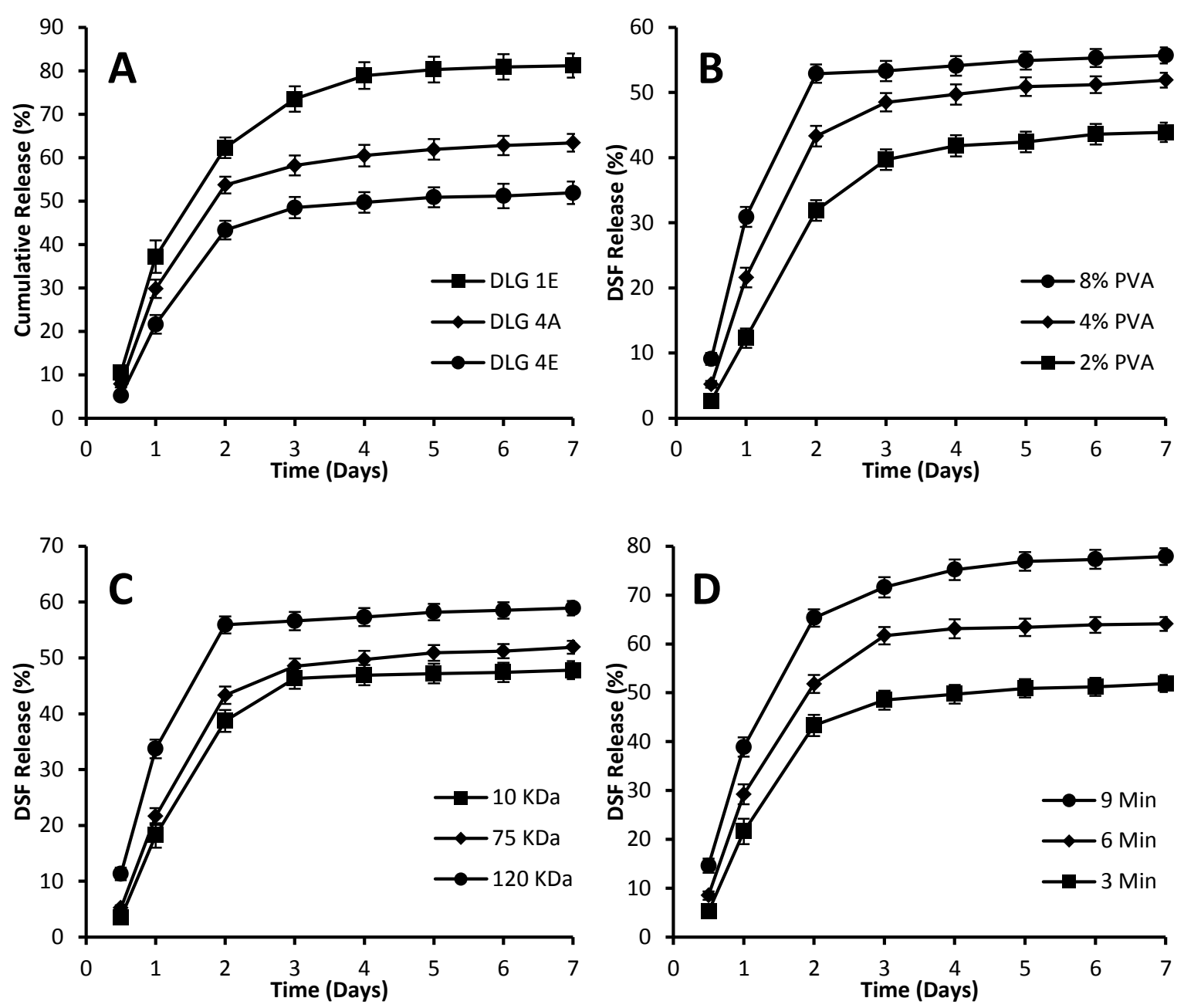
Figure 2
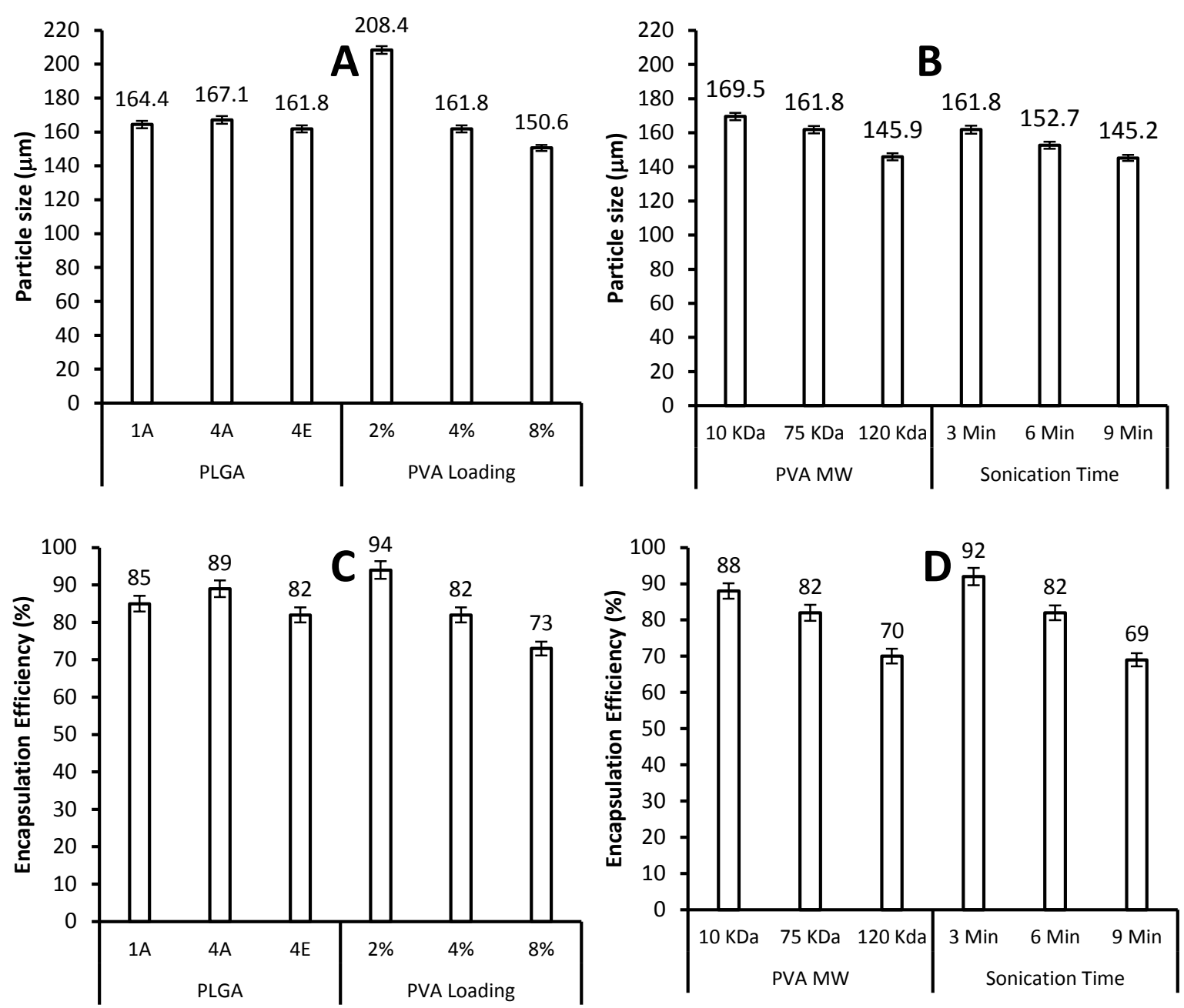
Figure 1

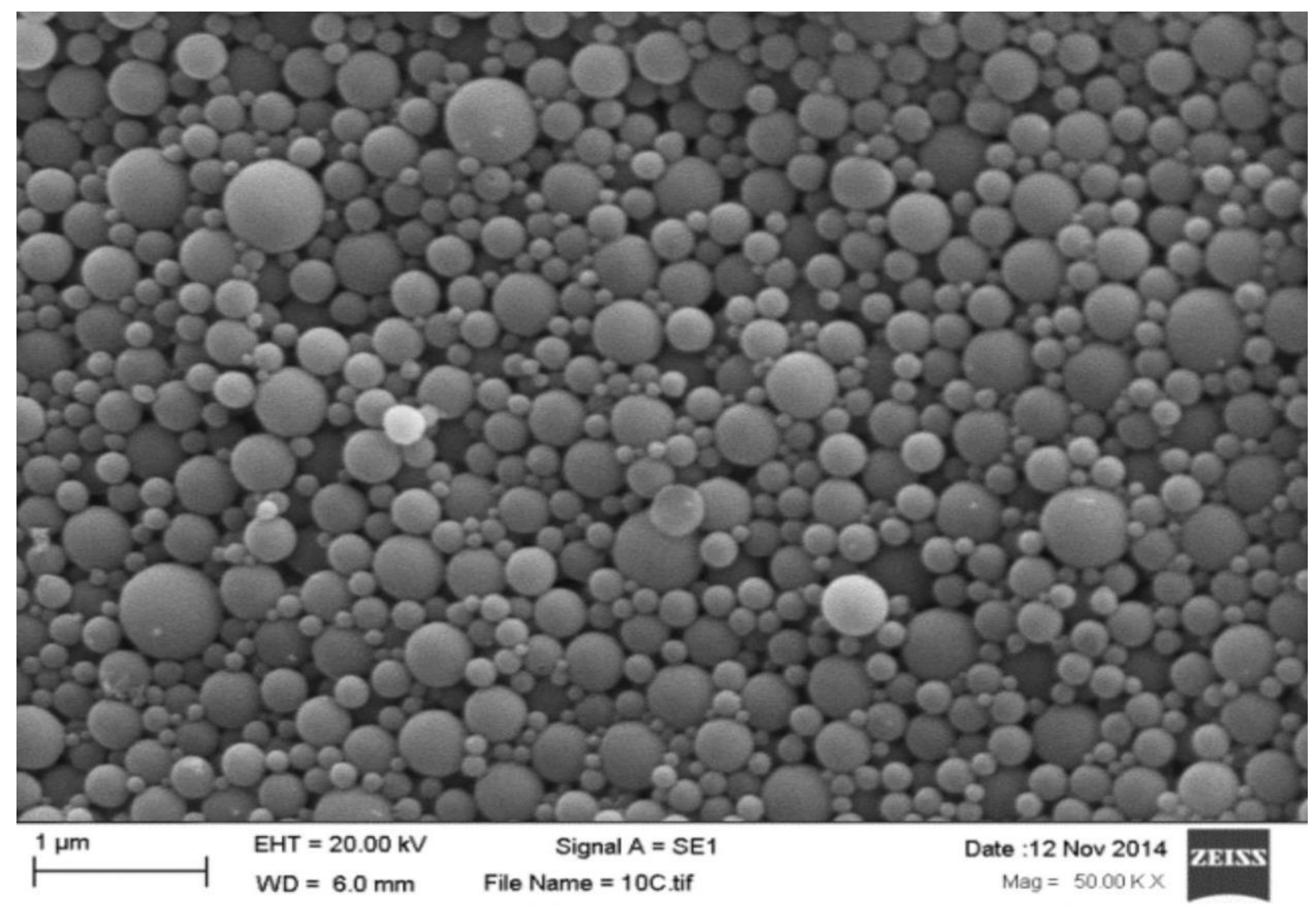

